artículos no derivados de proyectos de investigación 

CUAD. CONTAB. / BOCOTÁ, COLOMBIA, 15 (39): 831-852 / NÚMERO ESPECIAL 2014 / 831

\section{Costos indirectos de fabricación: propuesta para su tratamiento ante los cambios normativos que enfrenta Colombia}

doi: 10.11144/Javeriana.cc15-39.cifp

\section{Belky Esperanza Gutiérrez-Castañeda}

Contadora pública, Universidad Libre, Bogotá. Maestría en ciencias contables, Universidad de São Paulo, USP, Brasil. Doctorado en ciencias contables, Universidad de São Paulo, USP, Brasil. Profesora investigadora, Departamento de Ciencias Contables, Universidad de Antioquia.

Correos electrónicos: belkybw@gmail.com, belky.gutierrez@udea.edu.co

\section{María Isabel Duque-Roldán}

Contadora pública, Universidad de Antioquia. Especialista en revisoría fiscal, Universidad de Antioquia. Especialista en didáctica universitaria, Universidad de Antioquia. Maestría en ciencia política, Universidad de Antioquia. Profesora investigadora, Departamento de Ciencias Contables, Universidad de Antioquia.

Correos electrónicos: duqueroldan@gmail.com, isabel.duque@udea.edu.co 
Resumen La aplicación de los estándares internacionales de información financiera en Colombia, específicamente la NIC 2, implica un cambio sustancial en la forma como se clasifican y distribuyen los costos indirectos a los productos, por varias situaciones: 1) la definición de la base de actividad y el nivel de operación para el cálculo de la tasa de asignación de costos a los productos, 2) la determinación del comportamiento (fijo-variable) y 3 ) el tratamiento contable de las variaciones entre el valor predeterminado y el real. El siguiente estudio de caso aplicado a una empresa manufacturera que utiliza costeo tradicional, aprovecha herramientas estadísticas para dar solución al problema antes descrito: por medio de modelos de regresión lineal-multivariada se identifica la base que mayor relación tiene con los Costos Indirectos de Fabricación, CIF; y con técnicas de análisis de desviación se determina el comportamiento fijovariable. Se concluye que las bases de asignación analizadas por separado presentan un bajo nivel de significancia y un bajo $\mathrm{R}^{2}$ pero al combinarlas se obtiene un mejor resultado; así mismo, al analizar el comportamiento de los costos, se recomienda que sean catalogados en el grupo de fijos pues no es factible para ellos hallar una tasa unitaria que esté en función de la producción; también se describe el tratamiento que debe darse a las variaciones.

Palabras clave tasas predeterminadas de CIF; costos fijos; costos variables; bases de asignación de costos; tratamiento de las variaciones; NIC 2

\section{Código JEL M 41}

\section{Indirect Manufacturing Costs: A Proposal for their Treatment toward the Ongoing Regulatory Changes in Colombia}

\footnotetext{
Abstract The application of the international financial reporting standards in Colombia, specifically the NIC 2, imply a substantial change in the way indirect costs for products are classified and distributed due to a variety of reasons: 1) the definition of the activity base and the ope-
}

ration level for the calculation of the cost assignment rate for products; 2) determination of behavior (fixed-variable); and 3) the accounting treatment of the variations between the predetermined and real values. This case study, applied to a manufacturing company using traditional costing, uses statistical tools to solve the aforementioned problem: by means of multivariable-linear regression models we identify the basis that is most related to the Indirect Manufacturing Costs (CIF); using deviation analysis techniques we determined the fixed-variable behavior. We concluded that the assignment basis analyzed separately show a low significance level and a low $\mathrm{R}^{2}$, but lead to a better result when combined. Likewise, at the moment of analyzing cost behavior, we recommend that they are catalogued in the fixed group, as it is not feasible to find a unit rate in function of production for them; we also describe the treatment to be given for variations.

Keywords predetermined CIF rates; fixed costs; variable costs; cost assignment basis; treatment for variations; NIC 2

\section{Custos indiretos de fabricação: proposta para seu tratamento perante as alterações normativas que a Colômbia enfrenta}

Resumo A aplicação dos padrões internacionais de informação financeira na Colômbia, especificamente a IAS 2 , acarreta uma mudança substancial na forma como os custos indiretos são classificados e distribuídos aos produtos, por diferentes situações: 1) a definição da base de atividade e o nível de operação para o cálculo da taxa de alocação de custos aos produtos, 2) a determinação do comportamento (fixo-variável) e 3) o tratamento contábil das variações entre o valor predeterminado e o real. O seguinte estudo de caso aplicado numa empresa manufatureira que utiliza custeio tradicional, aproveita ferramentas estadísticas para dar solução ao problema descrito acima: através de modelos de regressão linear-multivariada é identificada a base que 


\section{COSTOS INDIRECTOS DE FABRICACIÓN: PROPUESTA PARA SU TRATAMIENTO / B. GUTIÉRREZ, M. DUQUE / 833}

tem maior relação com os Custos Indiretos de Fabricação, CIF; e com técnicas de análise de deviação determina-se o comportamento fixo-variável. Conclui-se que as bases de alocação analisadas por separado apresentam baixo nível de significância e baixo $\mathrm{R}^{2}$ mais quando combinadas obtém-se melhor resultado; da mesma forma, ao analisar o comportamento dos custos, recomenda-se que sejam catalogados no grupo de fixos pois não é factível para eles achar uma taxa unitária que esteja em função da produção; mesmo descreve-se o tratamento a se dar às variações.

Palavras-chave taxas predeterminadas de CIF; custos fixos; custos variáveis; bases de alocação de custos; tratamento das variações; IAS 2

\section{Introducción}

El proceso de convergencia a estándares internacionales de información financiera que se viene realizando en Colombia, involucra un análisis profundo sobre sus implicaciones para la información contable y de costos que preparan las empresas. En el tema específico de la aplicación de la NIC 2, hay cambios sustanciales que aún no han sido suficientemente analizados, sobre todo en lo que tiene qué ver con la separación de los costos indirectos en fijos y variables para su distribución a los productos o servicios, la definición de las bases de actividad que serán utilizadas para el cálculo de las tasas de Costos Indirectos de Fabricación - CIF-y el manejo contable de las variaciones que se pueden presentar entre el nivel de operación presupuestado y el realmente logrado.

En Colombia, la normatividad que actualmente rige las operaciones contables es muy limitada en lo que tiene qué ver con los procedimientos que deben llevarse a cabo para el cálculo de los costos de productos o servicios. El artículo 63 del Decreto 2649 de 1993, por el cual se reglamenta la contabilidad en general y se expiden los principios o normas de contabilidad generalmente aceptados en Colombia, se limita a señalar que en el costo del inventario deben incluirse los costos directos e indirectos necesarios para ponerlos en condiciones de utilización o venta, pero no se dice nada sobre la forma en que debe realizarse ese procedimiento, ni se definen los métodos permitidos para el cálculo de las tasas de costos indirectos; tampoco se plantea el manejo que debe hacerse de las variaciones que puedan presentarse por cambios en los niveles de producción o en las erogaciones realizadas.

Esta falta de reglas de juego claras ha hecho que las empresas apliquen los criterios que consideran más razonables. Por ello, como base de actividad, utilizan la de más fácil cálculo o la que consideran más acertada (horas/hombre, unidades producidas, horas/máquina, etc.) pero sin un soporte claro de la decisión; del mismo modo, aplican criterios subjetivos para separar los costos en fijos y variables y ni qué hablar del manejo que se les da a las variaciones entre los costos reales y los aplicados a la producción, pues muchas empresas sin un análisis claro toman la decisión de llevarlas a los resultados del período o, por el contrario, prorratearlas entre los inventarios y el costo de ventas.

Por tanto, ante un cambio normativo como el que viene experimentando el país, es necesario aportar elementos de juicio que permitan a las empresas: 1) mejorar sus procesos de cálculo y separación de costos indirectos, de definición de bases de actividad y niveles de operación, 
y de manejo contable de las variaciones y 2) adecuar sus sistemas de información a los nuevos requerimientos que imponen los estándares internacionales de información financiera NIIF, todo esto en el elemento del costo más complejo de manejar: los CIF. Para ello, se hace necesario utilizar herramientas estadísticas que soporten la definición de políticas de costos en las empresas, que mejoren los resultados y los hagan más confiables para la planeación y la toma de decisiones.

\section{Separación y asignación de costos indirectos: análisis normativo}

Para entender el manejo contable que deben tener los CIF y la magnitud del cambio que Colombia está enfrentando, es importante analizar cómo se describe el tratamiento de los costos asociados al inventario en cuatro normativas diferentes: Colombia, Estados Unidos, España y el estándar internacional, haciendo énfasis en lo que tiene qué ver con los CIF, para definir los puntos clave del análisis a partir de esta comparación:

De la comparación de estas cuatro normas se pueden extraer las siguientes conclusiones que sustentarán el análisis del rubro costos indirectos, que se hará en los próximos apartados:

- La norma colombiana es bastante corta en la definición de los aspectos claves del cálculo del costo de los productos o servicios en comparación con las otras normas analizadas, lo que no permite ningún análisis sobre su alcance.
- El costo de un producto/servicio está compuesto por costos directos (como el material y la mano de obra) y de costos indirectos. Los costos directos tienen la particularidad de que pueden asignarse a los productos de forma clara, mientras que para los costos indirectos se requieren criterios o tasas de distribución.

- Los costos indirectos variables deben tener un valor unitario fácilmente medible y calculable, mientras que para el resto de costos indirectos (que llamaremos fijos) se requiere el cálculo de una tasa de distribución, la cual se obtiene con base en el nivel normal de utilización de los factores productivos.

- El nivel o capacidad normal de producción es aquel que tiene en cuenta el comportamiento histórico de la producción, por lo que no incluye actividades de mantenimiento o preparación de máquinas ni otros factores que puedan afectar la producción en períodos específicos.

- Si el nivel de producción real es inferior al normal se generará una variación por la no utilización de la capacidad que no puede distribuirse a los productos y debe tratarse como un gasto del período en el cual se generó.

- La norma española es la única que hace énfasis en varios aspectos muy importantes: la necesidad de que los criterios de distribución de los costos sean calculados de forma sistemática (por medio de un sistema de costos), que sean mantenidos en el tiempo y de llegar a cambiarse, se justifiquen suficientemente las razones de ese cambio; así mismo, es la única que habla de la necesidad de incluir en las revelaciones que hace la empresa, los criterios de costos utilizados. 


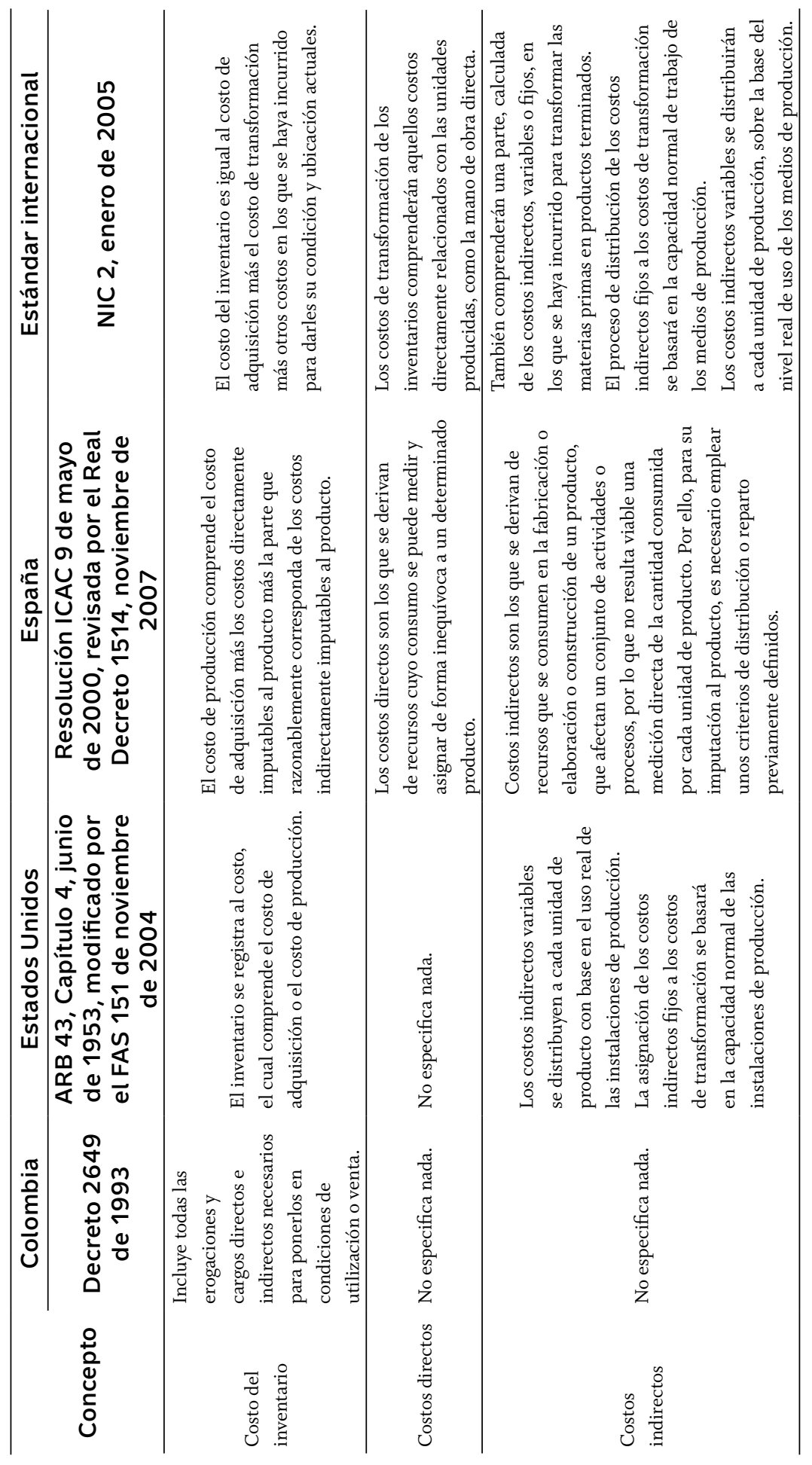


836 / VOL. 15 / NO. 39 / NÚMERO ESPECIAL 2014

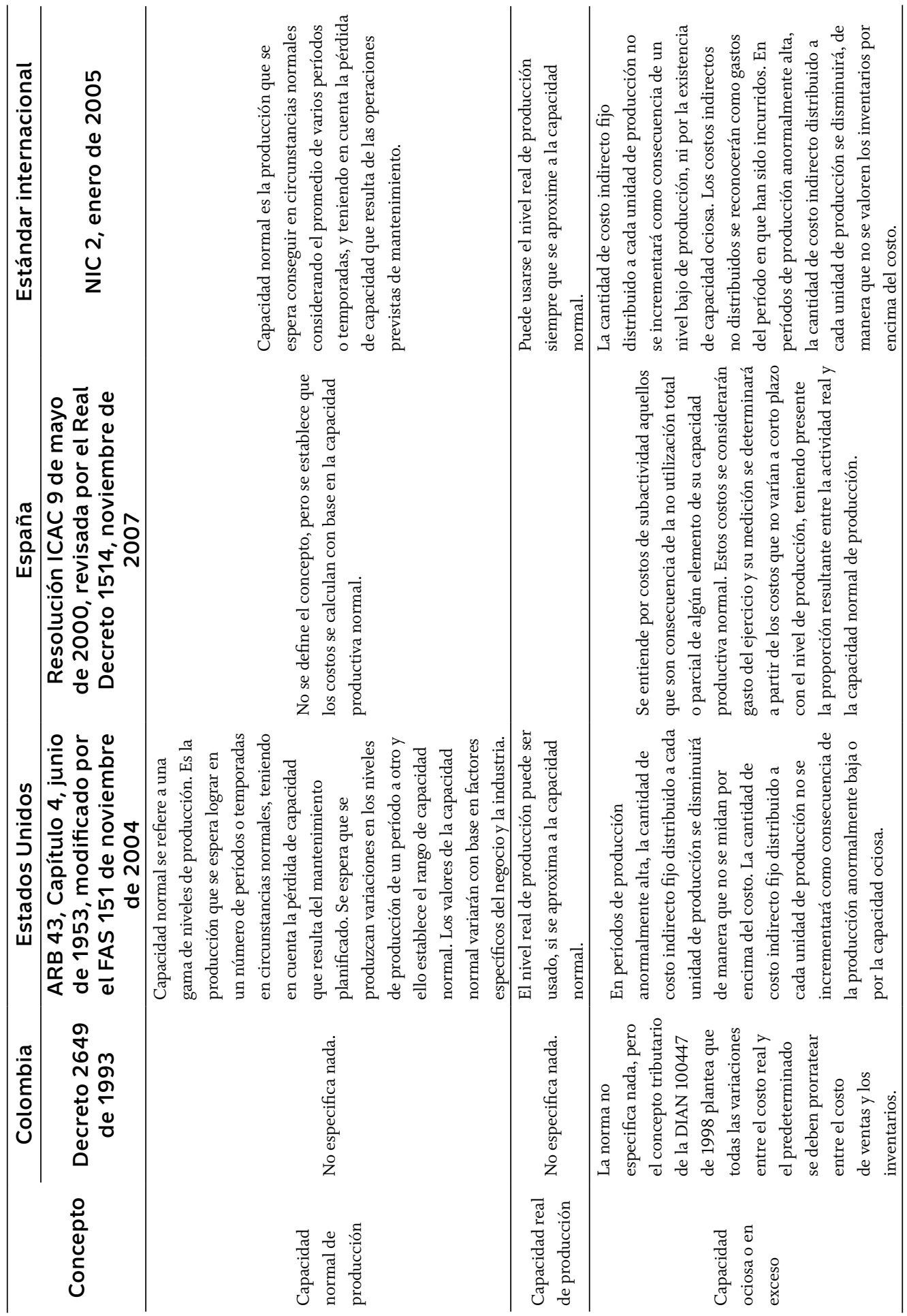


COSTOS INDIRECTOS DE FABRICACIÓN: PROPUESTA PARA SU TRATAMIENTO / B. GUTIÉRREZ, M. DUQUE / 837

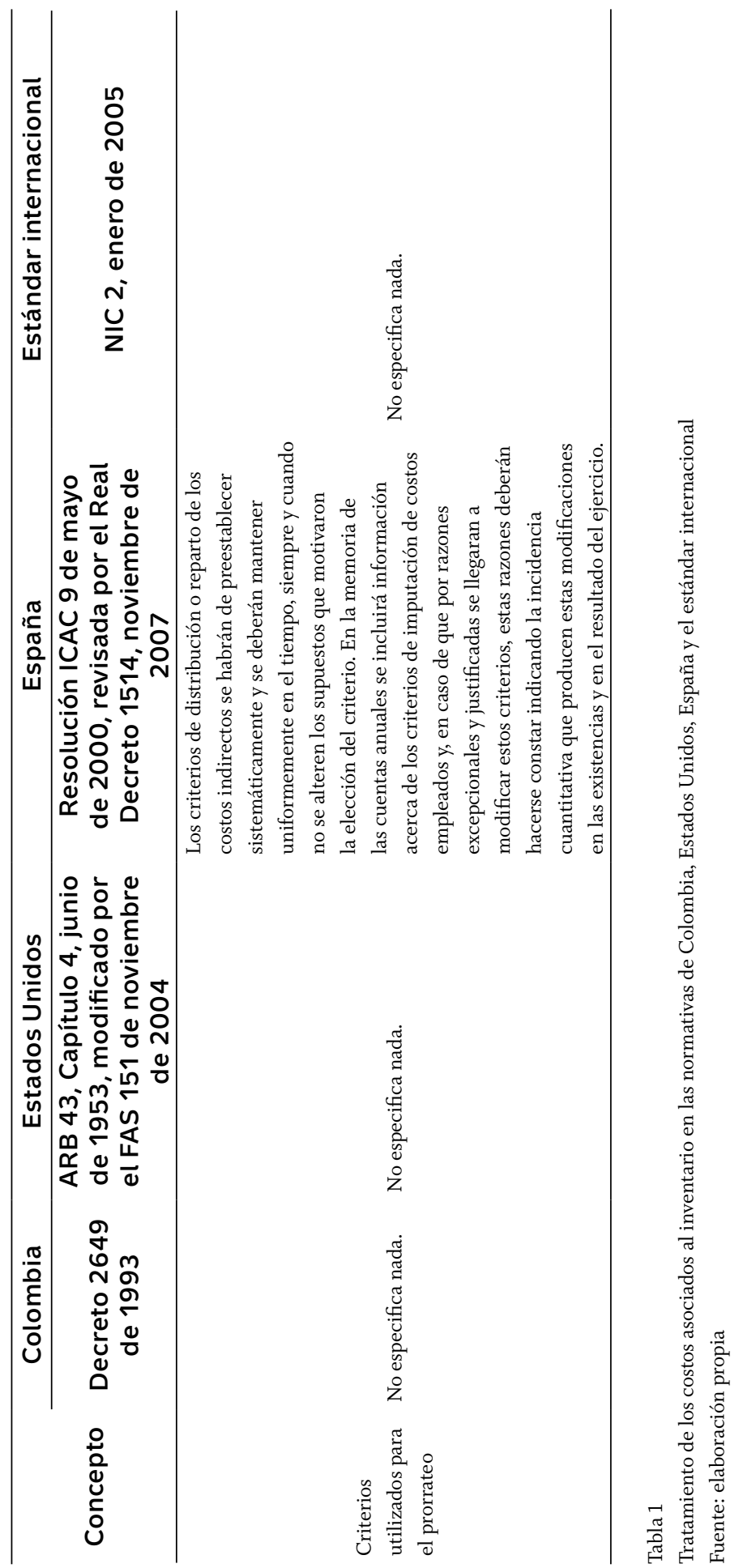


La comparación anterior permite concluir que Colombia no cuenta con procedimientos claros para la asignación de los costos indirectos a los productos o servicios, lo que deja abierta la posibilidad de manejos alternativos de acuerdo con la conveniencia de cada empresa, situación que resulta peligrosa por las siguientes circunstancias: a) los CIF vienen creciendo en importancia dentro de la estructura de costos de cualquier organización, pues incluye rubros como maquinaria, servicios públicos, personal indirecto, entre otros, que cada vez se hacen más importantes; b) la no utilización plena de estos CIF puede estar generando una capacidad ociosa que no se refleja en la información financiera y que, por tanto, conduce a fluctuaciones en los costos unitarios que dificultan la toma de decisiones; y c) al ponerse en práctica una nueva normativa (NIC 2) que sí presenta procedimientos claros para el manejo de los CIF, para muchas empresas ello implica grandes cambios en la forma de calcular y revelar este rubro, e incluso en sus resultados.

\section{El manejo de los costos indirectos: sus dificultades}

\subsection{Tasas de asignación de CIF}

Hay una amplia discusión frente a las metodologías de costeo más convenientes para cada tipo de empresa — tradicional o moderna-. He allí el dilema. Las principales críticas que se hacen a las metodologías tradicionales de costos, por parte de aquellos que promueven la utilización de las denominadas "nuevas técnicas de costeo" como el ABC o el throughput, se centran en la forma en la cual se distribuyen los costos a los productos/servicios, principalmente los costos indirectos no variables.

Para Joaquín Cuervo-Tafur, María Isabel Duque-Roldán y Jair Albeiro Osorio-Agudelo (2013), las metodologías tradicionales no formulan de manera objetiva la asignación del tercer elemento del costo. Siempre que los costos indirectos se apliquen sobre una base predeterminada, pueden encontrarse inconsistencias tanto en el numerador como en el denominador de la tasa: el presupuesto de costos indirectos y el nivel de operación presupuestado respectivamente; el primero, porque las empresas generalmente tienen que tolerar diferencias sustanciales entre lo presupuestado y lo ejecutado y el segundo, porque las expectativas realistas nunca han sido la mayor cualidad de los pronosticadores. Sin decir nada de la base de actividad que, por lo general, la que se selecciona no es la más razonable; adicionalmente, al aplicar una sola tasa de asignación los costos indirectos se asignan a todos los productos sin importar si realmente son consumidos por ellos. En síntesis, lo que se asigna al producto como costo indirecto no es real, como se muestra en el gráfico 1:

Para Eduardo Bendersky (2002), los sistemas tradicionales de costos basan el proceso de costeo en el producto, los costos se remiten al producto porque se presupone que cada elemento del producto consume los recursos en proporción al volumen producido. Por tanto, los atributos de volumen de producto, como el número de horas de mano de obra directa, horas/máquina, cantidad de materiales, entre otros, se utilizan como direccionadores para asignar los costos indirectos. Pero esta forma de 
COSTOS INDIRECTOS DE FABRICACIÓN: PROPUESTA PARA SU TRATAMIENTO / B. GUTIÉRREZ, M. DUQUE / 839

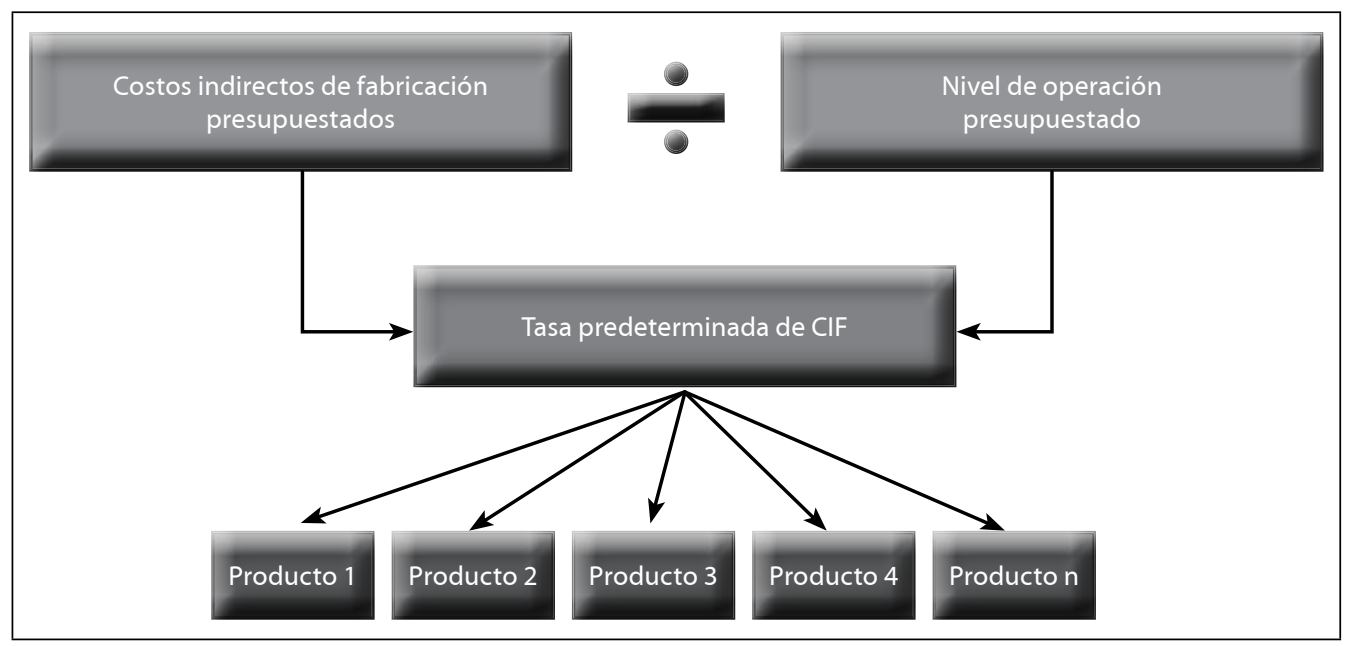

\section{Gráfico 1}

Procedimiento para la distribución de costos indirectos

Fuente: tomado de Joaquín Cuervo-Tafur, María Isabel Duque-Roldán, Jair Albeiro Osorio-Agudelo (2013). Costeo Basado en Actividades, ABC. Gestión de Costos Basada en Actividades, ABM

definición de las bases de distribución de costos resulta problemática. Para Charles T. Horngren, Srikant M. Datar y George Foster (2007), ante la dificultad de saber qué base de distribución se aplica en el prorrateo de costos indirectos, se opta por tomar la que se considere más representativa, que comúnmente son las horas de producción o los artículos fabricados. Según Alfredo Romero-Ceceña (1996), esto distorsiona sistemáticamente el costo de los productos e introduce todo tipo de subsidios entre ellos, pues muchas empresas utilizan una única tasa de prorrateo de costos indirectos de fabricación para toda la planta, la cual por asignar la misma cantidad de costos indirectos de fabricación a cada tipo de producto, falla en reconocer las diferencias en la atención u esfuerzo requeridos en la producción.

H. Thomas Johnson y Robert S. Kaplan (1988) también llaman la atención sobre las distribuciones arbitrarias de costos y el peligro que estas representan en la toma de decisiones pues pueden conducir a errores en materia de precios, fuentes de suministro, mezcla de productos y respuestas a productos rivales. En el mismo sentido, Leonel Arias-Montoya, Liliana Margarita Portilla de Arias y Sergio Augusto Fernández-Henao (2010) señalan que si la distribución de CIF no se realiza de manera apropiada, trae como consecuencia asignarle un mayor valor unitario a unos productos y menor a otros, lo que repercute negativamente en la rentabilidad de la empresa, porque unos productos subsidian a otros y no se tiene una información confiable para la toma de decisiones.

Pese a los reiterados señalamientos sobre el riesgo que se corre en el cálculo de costos unitarios en una empresa multiproducto con la utilización de una sola base de asignación, como lo hacen las metodologías tradicionales 
de costos, muchas empresas en Colombia y en el mundo aplican esta política, tal vez por facilidad, por el software de costos que utilizan o por desconocimiento. De acuerdo con las encuestas de prácticas globales en contabilidad de costos, disponibles en Charles T. Horngren, Srikant M. Datar y George Foster (2007), utilizar una o pocas bases de distribución para prorratear costos indirectos sigue siendo una práctica frecuente en las empresas, sobre todo del sector manufacturero. Esta encuesta muestra que las empresas prefieren el criterio de horas/hombre con los siguientes porcentajes: $84 \%$ en Nueva Zelanda, 78\% en Reino Unido, 73\% en Australia, 68\% en Japón, 62\% en Estados Unidos y $52 \%$ en Irlanda, aunque algunas empresas reconocen que utilizan otras bases.

\subsection{Separación de costos en fijos y variables}

Diferentes autores han abordado el tema del comportamiento de los costos. Enrique Nicolás Cartier (2002), citando a Erich Schneider, afirma que la subdivisión de los costos en fijos y variables es el resultado de examinar la relación funcional entre los costes y una o más variables independientes. $\mathrm{Al}$ hablar de variables independientes, hace referencia a los criterios tradicionalmente utilizados para distribuir costos: la actividad, la producción, la ocupación y la productividad. Este autor concluye que en el análisis de los costos entra solamente la cantidad de producción como variable independiente primaria.

Para Jorge Ignacio Artigas y Javier Eduardo Zayún (2013), los costos son relativos y es necesario separarlos en fijos y variables y distribuir- los de acuerdo con un nivel de operación que tenga en cuenta aspectos como los cambios en la capacidad de producción a corto y mediano plazo y la controlabilidad de este nivel.

Por el contrario, Alfredo Kaplan y Amaro Yardín (2013) llaman la atención a los teóricos para que abandonen la clásica separación de los costos en fijos y variables y proponen utilizar el concepto de "sensibilidad", para aceptar que el comportamiento de los costos frente a cambios del nivel de actividad no puede definirse con solamente dos clases de costos, porque ellos pueden estar vinculados a distintos factores que afecten de muchas maneras su comportamiento.

Estudios como el de Felipe Dantas Cassimiro da Silva, Marco Túlio de Castro Vasconcelos, Alexandre César Batista da Silva y Sebastião Marcos Campelo (2006) o el de Sérgio de Iudícibus (1989), han abordado el análisis del comportamiento de los costos (fijos y variables) utilizando como base de análisis las horas de mano de obra directa empleadas como principal inductor de costos y herramientas como el análisis de regresión para determinar la porción fija y variable.

\subsection{Manejo de las variaciones de CIF}

Frente al manejo de las variaciones de CIF, en la literatura de costos es muy frecuente encontrar diferentes alternativas:

- Cancelarlas contra el costo de ventas o los resultados.

- Prorratearlas entre los inventarios de productos en proceso y producto terminado y el costo de ventas.

- Asignar algunas de las variaciones directamente a resultados y prorratear el resto entre los inventarios y el costo de ventas. 
Para Letricia Gayle Rayburn (1999), cuando la variación no es significativa frente al costo total de los productos vendidos, al ingreso total de operación o respecto de alguna otra prueba de importancia relativa, se asigna la cantidad sobreaplicada o subaplicada a la cuenta de costo de productos vendidos; por el contrario, los contadores distribuyen las variaciones entre la producción en proceso, los productos terminados y el costo de ventas basándose en la proporción relativa de las unidades vendidas, terminadas y en proceso, cuando la cantidad en cuestión afecta de manera sustancial las valuaciones de los inventarios de la empresa.

El método escogido para la distribución de las variaciones deberá depender de la importancia de las cantidades que intervienen. Si la variación no es significativa, se podrían traspasar a la cuenta de costo de ventas. Sin embargo, cuando las variaciones se originan por alguna irregularidad imprevista, su distribución es el procedimiento más adecuado. El método más aconsejable a seguir es juzgar cada variación individualmente y tomar una decisión basada en su causa (Océano Grupo Editorial, 2002).

Aunque la teoría de costos siempre recomienda un análisis previo a la decisión sobre el manejo de las variaciones, en Colombia el concepto 100447 de 1998 de la DIAN recomienda que todas las variaciones sean prorrateadas entre los inventarios y el costo por lo que es normal encontrar que aquellas empresas que utilizan sistemas predeterminados de costos, al finalizar el período recalculan el costo de sus productos incluyendo la variación, sin mediar análisis de sus causas.
No se encontraron estudios sobre la escogencia de la mejor base de actividad para el cálculo de las tasas de CIF.

\section{Metodología}

Para demostrar la forma de definir la base de actividad más adecuada para calcular la tasa de asignación de costos indirectos, se realizó un Estudio Caso en una empresa del sector manufacturero que utiliza un sistema de costeo por órdenes de fabricación y cuya base de costeo es estándar. Se analiza el comportamiento de los costos indirectos de fabricación (CIF) frente a dos posibles bases de actividad, que según la literatura y la experiencia de la empresa tienen una buena relación con los CIF: el Costo de Materia Prima (CMP) y las unidades (kilos) producidas (KP); no se utiliza el criterio de horas/hombre, porque el proceso productivo está basado en máquinas y porque la empresa no cuenta con un control estricto del tiempo que el personal efectivamente dedica a la función de la producción. La empresa históricamente ha utilizado el criterio de kilos producidos para distribuir los CIF, pero las variaciones entre el costo aplicado y el real suelen ser altas en cada período.

Se analizó y documentó el comportamiento histórico mensual de los costos indirectos consumidos por la empresa, durante el período comprendido entre agosto de 2012 y noviembre de 2013 y mediante un análisis de regresión simple y multivariada con la ayuda del software SPSS 21 se determinó como variable dependiente $Y$ el valor de los costos indirectos (CIF) y como variables indepen- 
dientes $X$ el costo del material directo (CMP)

y las unidades producidas en kilos (KP). El

objetivo de este análisis es determinar cuál de estas dos variables tiene mayor nivel de significancia frente a la variable dependiente; en este caso, los CIF.

$Y(C I F)=\beta_{0}+\beta_{1} \times(C M P)+\varepsilon_{i, j}$

Los Betas $(\beta)$ representan los coeficientes que determinan la relación lineal entre las variables; igualmente, ayudan a determinar cuál base (variables independientes) tiene más nivel de significancia. En nuestro estudio, no se presenta un análisis del valor $T$ de los coeficientes, ya que estos se utilizan para constatar una hipótesis propuesta y en este estudio, el objetivo no era evaluar una hipótesis, sino identificar la base de actividad que tuviera un mayor nivel de significancia frente a los CIF. Aunque reconocemos que el análisis de valor $T$ de coeficientes es utilizado para muestras de tamaño pequeño y la muestra utilizada en este estudio es de 16 datos (meses), su tamaño se considera adecuado teniendo en cuenta que los ciclos de costos son mensuales.

Aunque se toman los datos de una empresa específica, el análisis y la formulación estadística realizados pueden ser aplicables a cualquier empresa.

Para separar los costos en fijos y variables, en este trabajo se renunció a la utilización de modelos de regresión o de estimación lineal para determinar el comportamiento de los costos con base en un nivel de operación definido, dado que se partirá de las precisiones hechas por Alfredo Kaplan y Amaro Yardín (2013) en el sentido de que los costos indirectos tienen diferentes grados de sensibilidad; por tanto, se analizó el valor de los CIF de la empresa durante 16 meses y para este conjunto de datos se calcularon la desviación estándar, la media y el coeficiente de variación para determinar la heterogeneidad de los valores y con base en ello determinar si el comportamiento tiende a ser fijo o variable y luego proceder al cálculo de la tasa de distribución.

\section{Resultados}

Definición de la base de actividad: el procedimiento seguido para la definición de la base de actividad más indicada consiste en comparar la variable dependiente (CIF) con cada una de las variables independientes (CMP y KP).

Inicialmente, se realiza una regresión lineal simple entre las variables CIF y CMP obteniendo como resultado:

$Y(C I F)=2230666,884+114,572 \times(C M P)+\varepsilon$ $Y(C I F)=-4800320,959+, 057 \times(K P)+\varepsilon$

Se puede verificar, como se muestra en la tabla resumen 2 que hay una relación parcial entre la variable dependiente costo indirecto (CIF) y la variable independiente costo de materia prima (CMP) al correr el modelo de regresión lineal simple, lo que arroja un $\mathrm{R}^{2}(\mathrm{R}$ Cuadrado) de 55\%, lo que significa que solo se puede establecer una relación del 55\% entre el costo indirecto de fabricación registrado por la empresa en un período específico y el costo de la materia prima consumida en ese mismo período. 


\begin{tabular}{|c|c|c|c|c|c|c|c|c|c|c|}
\hline \multirow[b]{2}{*}{$\begin{array}{l}\frac{0}{0} \\
\frac{0}{0} \\
\sum\end{array}$} & \multirow[b]{2}{*}{$\simeq$} & \multirow{2}{*}{$\begin{array}{l}0 \\
\frac{0}{0} \\
\frac{0}{0} \\
\frac{0}{0} \\
己 \\
\simeq \\
\simeq\end{array}$} & \multirow{2}{*}{ 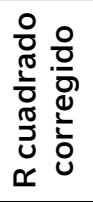 } & \multirow{2}{*}{ 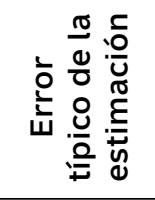 } & \multicolumn{5}{|c|}{ Estadísticos de cambio } & \multirow[b]{2}{*}{$\begin{array}{l}\text { Durbin- } \\
\text { Watson }\end{array}$} \\
\hline & & & & & $\begin{array}{c}\text { Cambio } \\
\text { en } \mathrm{R} \\
\text { cuadrado }\end{array}$ & $\begin{array}{c}\text { Cambio } \\
\text { en } F\end{array}$ & gl1 & gl2 & $\begin{array}{c}\text { Siguiente } \\
\text { cambio } \\
\text { en } F\end{array}$ & \\
\hline 1 &, $235^{\mathrm{a}}$ &, 55 &,- 12 & 9935407,412 & ,055 & ,816 & 1 & 14 & ,382 & 1,421 \\
\hline
\end{tabular}

Tabla 2

Resumen del modelo ${ }^{\mathrm{b}}$

a. Variables predictoras: (constante), CMP

b. Variable dependiente: CIF

Fuente: elaboración propia

Otra cosa sucede cuando se realiza la regresión lineal con la otra base de cálculo: kilos producidos (KP) (tabla 3), pues esta variable solo explica el comportamiento de la variable CIF en un $22 \%$, lo que resulta preocupante teniendo en cuenta que esta era la base utilizada por la empresa para la distribución de los CIF.
Inicialmente, se puede concluir que estas dos bases de análisis, que son utilizadas habitualmente en la práctica para la distribución de los costos indirectos (CIF), no representan en un alto porcentaje el comportamiento de los CIF de la empresa objeto de estudio.

\begin{tabular}{|c|c|c|c|c|c|c|c|c|c|c|}
\hline \multirow[b]{2}{*}{$\begin{array}{l}\frac{0}{0} \\
\frac{10}{0} \\
\sum\end{array}$} & \multirow[b]{2}{*}{$\propto$} & \multirow{2}{*}{$\begin{array}{l}0 \\
\frac{0}{0} \\
\frac{0}{0} \\
\frac{1}{0} \\
\frac{0}{2} \\
0 \\
\simeq\end{array}$} & \multirow{2}{*}{ 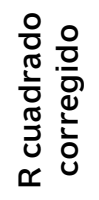 } & \multirow{2}{*}{ 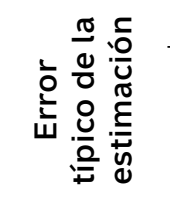 } & \multicolumn{5}{|c|}{ Estadísticos de cambio } & \multirow[b]{2}{*}{$\begin{array}{l}\text { Durbin } \\
\text { Watson }\end{array}$} \\
\hline & & & & & $\begin{array}{c}\text { Cambio } \\
\text { en R } \\
\text { cuadrado }\end{array}$ & $\begin{array}{c}\text { Cambio } \\
\text { en F }\end{array}$ & gl1 & $\mathrm{gl2}$ & $\begin{array}{c}\text { Siguiente } \\
\text { cambio } \\
\text { en F }\end{array}$ & \\
\hline 1 &, $148^{\mathrm{a}}$ & 22 &,- 48 & 10108709,707 & ,022 & ,312 & 1 & 14 &, 585 & 1,336 \\
\hline
\end{tabular}

Tabla 3

Resumen del modelo ${ }^{\mathrm{b}}$

a. Variables predictoras: (constante), KP

b. Variable dependiente: CIF

Fuente: elaboración propia

Además del análisis anterior, el pequeño poder explicativo de las variables se puede originar en el hecho de que los datos de la muestra presentan una elevada dispersión de los puntos en torno a la recta de regresión (gráfico 2). Una forma de solucionar este problema es considerar nuevas variables independientes en el modelo de regresión o considerar un estudio histórico más amplio.

Igualmente, al realizar el análisis gráfico por histograma de estas dos variables de mane- ra independiente, se identificó que tienen un comportamiento similar durante el período de estudio histórico, con una curva de frecuencia concentrada al lado izquierdo, lo que representa una "cola" más alargada a la derecha, es decir, la distribución es asimétrica a la derecha y presenta una asimetría positiva. En este caso, se tiene $\mathrm{Mo}<\mathrm{Md}<\overline{\mathrm{X}}$, así se puede concluir que los coeficientes son positivos en torno a la media. 


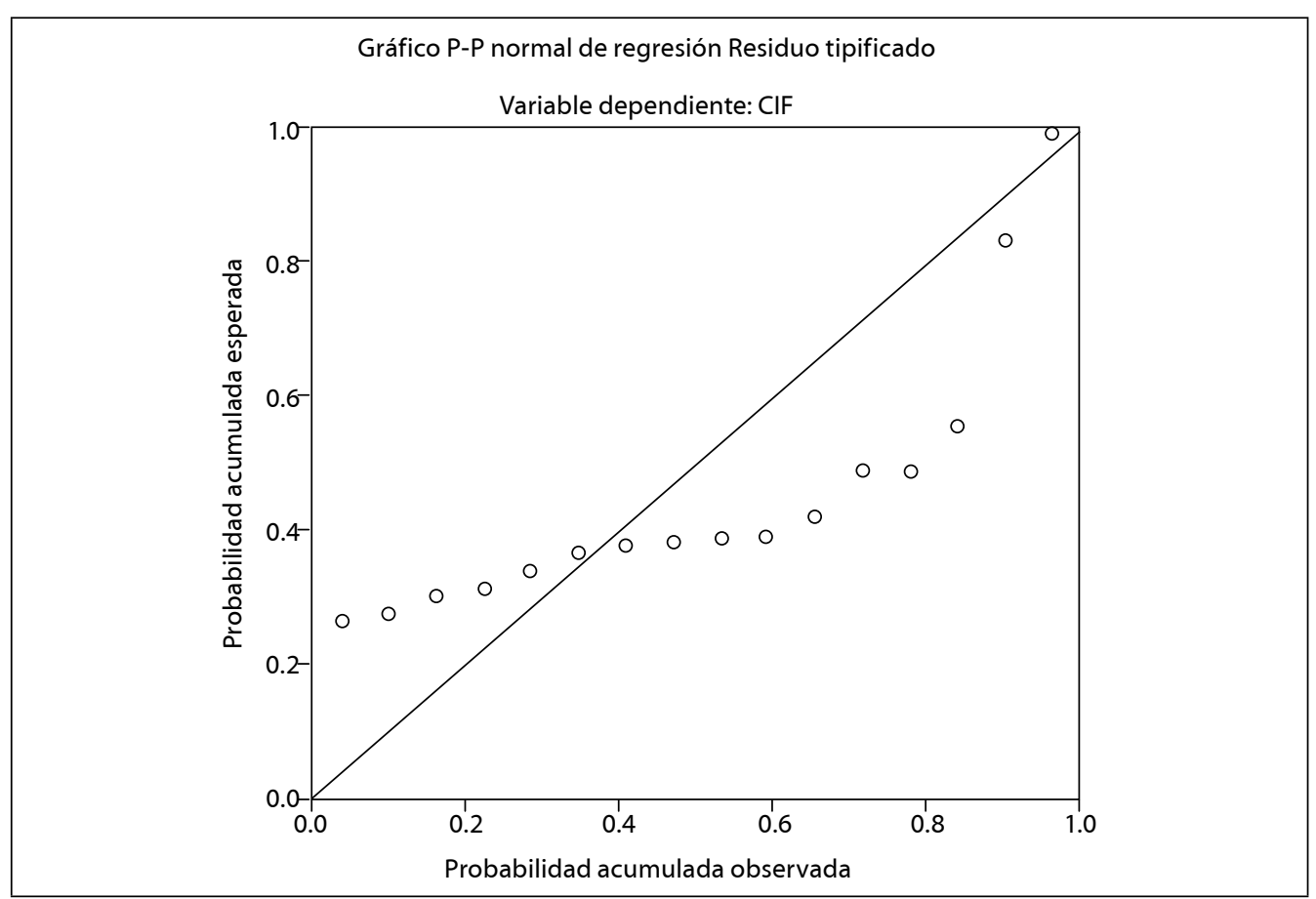

Gráfico 2

Dispersión

Fuente: elaboración propia

$\mathrm{Al}$ encontrar este poder explicativo tan bajo entre estas dos bases de asignación de costos estudiadas, se determinó realizar un modelo de regresión lineal multivariable (ecuación 4), para analizar el poder explicativo entre estas dos variables combinadas frente al CIF (tabla 4); así, se identificó que la combinación de estas dos bases determina un alto poder explicativo del comportamiento de la variable dependiente CIF, lo que indica que cerca del 95\% del comportamiento de la variable CIF se explican por las variables independientes en estudio cuando son combinadas.
Igualmente, para poder generalizar el comportamiento de estas dos bases de estudio frente al CIF se pueden realizar otros análisis que le ayudan a dar fuerza a las conclusiones presentadas en esta investigación como es el caso de los presupuestos de análisis de regresión lineal; específicamente, la ausencia de correlación serial en los residuos y de la multicolinearidad entre las variables independientes fueron testadas utilizando la estadística de Durbin-Watson (tabla 5).

$Y(C I F)=-101083,649+0,187 \times(C M P)+$ $-437,931 \times(K P)+\varepsilon$ 
COSTOS INDIRECTOS DE FABRICACIÓN: PROPUESTA PARA SU TRATAMIENTO / B. GUTIÉRREZ, M. DUQUE / 845

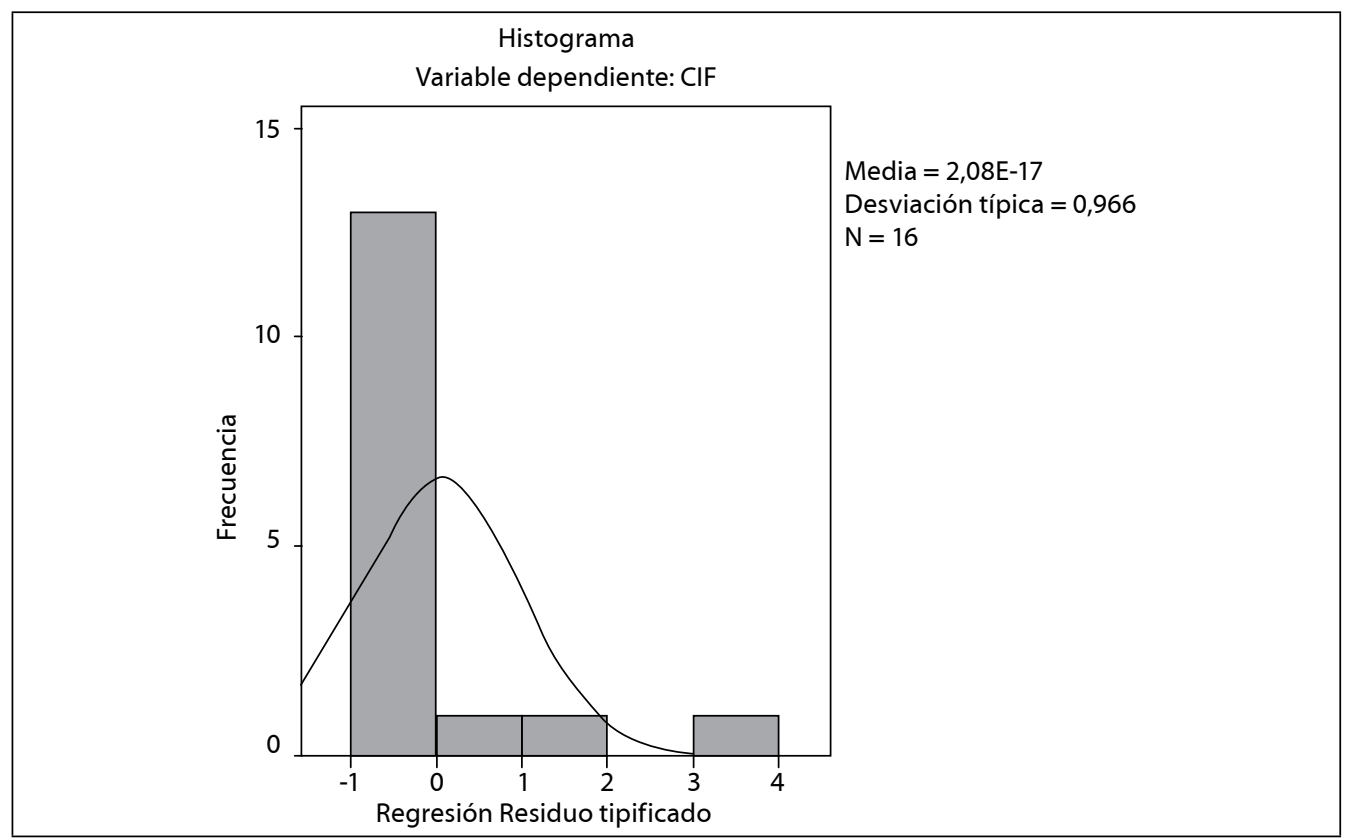

Gráfico 3

Histograma de frecuencia CMP-CIF

Fuente: elaboración propia

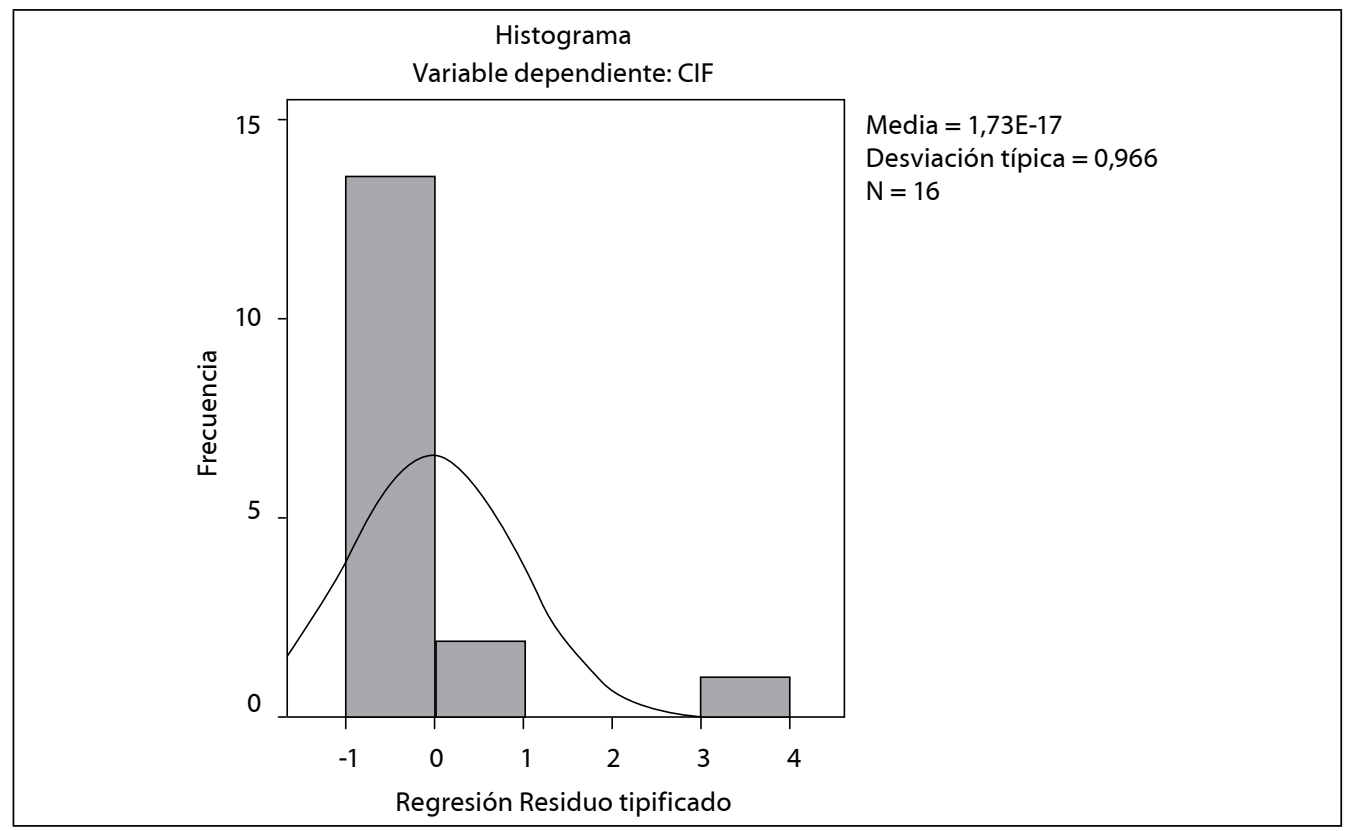

Gráfico 4

Histograma de frecuencia KP-CIF

Fuente: elaboración propia 


\begin{tabular}{|c|c|c|c|c|c|c|c|c|c|c|}
\hline \multirow[b]{2}{*}{$\begin{array}{l}\frac{0}{0} \\
\frac{0}{0} \\
\frac{0}{\Sigma}\end{array}$} & \multirow[b]{2}{*}{$\simeq$} & \multirow{2}{*}{ 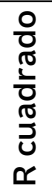 } & \multirow{2}{*}{ 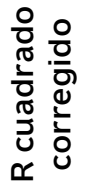 } & \multirow{2}{*}{ 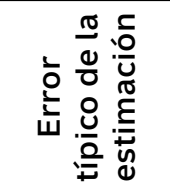 } & \multicolumn{5}{|c|}{ Estadísticos de cambio } & \multirow[b]{2}{*}{$\begin{array}{l}\text { Durbin- } \\
\text { Watson }\end{array}$} \\
\hline & & & & & $\begin{array}{c}\text { Cambio } \\
\text { en R } \\
\text { cuadrado }\end{array}$ & $\begin{array}{c}\text { Cambio } \\
\text { en } F\end{array}$ & gl1 & $g 12$ & $\begin{array}{c}\text { Siguiente } \\
\text { cambio } \\
\text { en F }\end{array}$ & \\
\hline 1 & $309^{\mathrm{a}}$ & ,95 &,- 44 & 10087560,991 & ,095 & ,686 & 2 & 13 &, 521 & 1,505 \\
\hline
\end{tabular}

Tabla 4

Resumen del modelo ${ }^{b}$

a. Variables predictoras: (constante), KP, CMP

b. Variable dependiente: CIF

Fuente: elaboración propia

\begin{tabular}{|c|c|c|c|c|}
\hline Presupuestos & Teste & Estadística & Siguiente t & $\begin{array}{l}\text { Evaluación (nivel de } \\
\text { significancia de 5\%) }\end{array}$ \\
\hline $\begin{array}{l}\text { Ausencia de correlación } \\
\text { serial en los residuos }\end{array}$ & Durbin-Watson & 1,505 & --- & \\
\hline $\begin{array}{l}\text { Multicolinearidad entre } \\
\text { variables independientes }\end{array}$ & $\begin{array}{l}\text { Máximo VIF/mínima } \\
\text { Tolerance }\end{array}$ & $7.888 / 0,12$ & -.-- & \\
\hline
\end{tabular}

Tabla 5

Evaluación de los presupuestos de regresión lineal Fuente: elaboración propia

Comportamiento de los costos: en este aspecto, se pretende determinar la homogeneidad de los CIF durante los diferentes períodos, para establecer si se separan en fijos y variables y se calculan tasas independientes para cada grupo. Para ello, se calcularon tres valores importantes: la media, la desviación estándar y el coeficiente de variación que permite medir con mayor confiabilidad la variabilidad de los datos. Los resultados son los siguientes:

\begin{tabular}{cc}
\hline Meses & Valor total CIF \\
\hline 1 & 56.624 .266 \\
\hline 2 & 43.763 .361 \\
\hline 3 & 45.588 .693 \\
\hline 4 & 42.747 .211 \\
\hline 5 & 40.778 .669 \\
\hline 6 & 34.193 .082 \\
\hline
\end{tabular}

\begin{tabular}{cc}
\hline Meses & Valor total CIF \\
\hline 7 & 43.412 .309 \\
\hline $\mathbf{8}$ & 42.396 .945 \\
\hline 10 & 44.447 .288 \\
\hline 11 & 42.086 .608 \\
\hline 12 & 43.860 .263 \\
\hline 13 & 43.799 .271 \\
\hline 14 & 44.933 .864 \\
\hline 15 & 42.401 .098 \\
\hline 16 & 42.411 .376 \\
\hline Desviación & 45.226 .025 \\
\hline Media & 4.340 .433 \\
\hline Coeficiente de variación & 41.353 .574 \\
\hline
\end{tabular}

Tabla 6

Resultados: media, desviación estándar y coeficiente de variación

Fuente: elaboración propia 
Con un coeficiente de variación de $10 \%$, se concluye que los datos son relativamente homogéneos y que, para el cálculo de las tasas de CIF, es preferible considerarlos no variables (fijos). El análisis se hizo para cada componente de los CIF y en forma global. En caso de que un componente hubiera dado un coeficiente alto, se clasificaría como variable. Adicionalmente, ninguno de los conceptos que conforman el rubro CIF permite el cálculo de una tasa unitaria de consumo, lo que refuerza la decisión de manejarlos como fijos para el cálculo de la tasa.

\section{Discusión de los resultados}

Los resultados que arroja la investigación nos permiten concluir que si la empresa solo tiene información sobre estas dos posibles bases de actividad para calcular las tasas de aplicación de los CIF, cualquiera de ellas utilizada de forma independiente arrojaría unos CIF aplicados poco confiables, lo que generaría variaciones altas; sin embargo, si se combinan las dos bases al momento de determinar el valor de los CIF aplicados, el resultado será mucho más acertado. A continuación, presentamos un ejemplo que describe la situación.

Se presentan datos ficticios para calcular la tasa de CIF; la primera columna indica el número de períodos históricos que se tuvieron en cuenta para el análisis y cálculo de la tasa predeterminada; en este caso, corresponde a 10 meses; la segunda columna muestra el valor de CIF registrados en la información contable de la empresa en cada uno de los 10 meses; la columna 3 muestra el costo de la materia prima consumida en cada uno de los períodos; la columna 4 presenta el dato de los kilos producidos en cada período y las columnas 5 y 6 muestran el cálculo de la tasa de aplicación de los CIF de acuerdo con cada una de las bases y que resulta de la siguiente fórmula: CIF/CMP y CIF/KP. La última fila muestra el presupuesto para el próximo período y de acuerdo con ello, las tasas de CIF a aplicar.

\begin{tabular}{cccccc}
\hline Período & CIF & CMP & KP & Tasa de CIF/CMP & Tasa de CIF/KP \\
\hline $\mathbf{1}$ & $\$ 52.000 .000$ & $\$ 89.000 .000$ & $52.000 \mathrm{~K}$ & 0,58 & $\$ 1.000,00 / \mathrm{K}$ \\
\hline $\mathbf{2}$ & $\$ 53.000 .000$ & $\$ 91.000 .000$ & $50.000 \mathrm{~K}$ & 0,58 & $\$ 1.060,00 / \mathrm{K}$ \\
\hline $\mathbf{3}$ & $\$ 63.000 .000$ & $\$ 92.000 .000$ & $53.000 \mathrm{~K}$ & 0,68 & $\$ 1.188,68 / \mathrm{K}$ \\
\hline $\mathbf{4}$ & $\$ 52.000 .000$ & $\$ 100.000 .000$ & $58.000 \mathrm{~K}$ & 0,52 & $\$ 896,55 / \mathrm{K}$ \\
\hline $\mathbf{5}$ & $\$ 61.000 .000$ & $\$ 85.000 .000$ & $50.000 \mathrm{~K}$ & 0,72 & $\$ 1.220,00 / \mathrm{K}$ \\
\hline $\mathbf{6}$ & $\$ 59.000 .000$ & $\$ 84.000 .000$ & $48.000 \mathrm{~K}$ & 0,70 & $\$ 1.229,17 / \mathrm{K}$ \\
\hline $\mathbf{7}$ & $\$ 58.000 .000$ & $\$ 96.000 .000$ & $52.000 \mathrm{~K}$ & 0,60 & $\$ 1.115,38 / \mathrm{K}$ \\
\hline $\mathbf{8}$ & $\$ 57.000 .000$ & $\$ 85.000 .000$ & $45.000 \mathrm{~K}$ & 0,67 & $\$ 1.266,67 / \mathrm{K}$ \\
\hline $\mathbf{9}$ & $\$ 56.000 .000$ & $\$ 90.000 .000$ & $55.000 \mathrm{~K}$ & 0,62 & $\$ 1.018,18 / \mathrm{K}$ \\
\hline $\mathbf{1 0}$ & $\$ 68.000 .000$ & $\$ 89.000 .000$ & $56.000 \mathrm{~K}$ & 0,76 & $\$ 1.214,29 / \mathrm{K}$ \\
\hline Presupuesto & $\$ 65.000 .000$ & $\$ 90.100 .000$ & $51.900 \mathrm{~K}$ & 0,7214 & $\$ 1.252,4084 / \mathrm{K}$ \\
\hline
\end{tabular}

Tabla 7

Tasas de CIF calculadas de manera independiente

Fuente: elaboración propia 
En el período para el cual se habían realizado los presupuestos se procesaron 10 órdenes de producción con la siguiente información:

\begin{tabular}{|c|c|c|c|c|}
\hline $\begin{array}{l}\text { Número } \\
\text { de orden }\end{array}$ & $\begin{array}{l}\text { Kilos } \\
\text { producidos } \\
(\mathrm{KP})\end{array}$ & $\begin{array}{c}\text { Costo materia } \\
\text { prima consumida } \\
(\mathrm{MP})\end{array}$ & CIF aplicados/KP & CIF aplicados/CMP \\
\hline 1 & 2.000 & 3.200 .000 & 2.504 .817 & $2.308 .546,06$ \\
\hline 2 & 10.000 & 13.000 .000 & 12.524 .085 & $9.378 .468,37$ \\
\hline 3 & 12.000 & 15.500 .000 & 15.028 .902 & $11.182 .019,98$ \\
\hline 4 & 5.000 & 6.800 .000 & 6.262 .042 & $4.905 .660,38$ \\
\hline 5 & 7.000 & 9.100 .000 & 8.766 .859 & $6.564 .927,86$ \\
\hline 6 & 6.000 & 7.800 .000 & 7.514 .451 & $5.627 .081,02$ \\
\hline 7 & 8.000 & 10.100 .000 & 10.019 .268 & $7.286 .348,50$ \\
\hline 8 & 5.000 & 7.100 .000 & 6.262 .042 & $5.122 .086,57$ \\
\hline 9 & 6.000 & 7.500 .000 & 7.514 .451 & $5.410 .654,83$ \\
\hline Total & 61.000 & 80.100 .000 & 76.396 .917 & $57.785 .794,00$ \\
\hline
\end{tabular}

Tabla 8

Aplicación de CIF a la producción real

Fuente: elaboración propia

Los CIF aplicados con cada una de las bases se obtienen de multiplicar la tasa hallada en la tabla 8 por el valor real de cada orden. Para la orden 1 sería:

- Con la base kilos producidos: 2.000 kilos * $\$ 1.252,4084 / \mathrm{K}=\$ 2.504 .820$

- Con la base de costo materia prima:

$\$ 3.200 .000 * 0.7214=\$ 2.308 .546$

$\mathrm{Al}$ aplicar cada una de las bases de manera independiente, se encuentra que con el criterio de kilos producidos se tendrían unos CIF aplicados de $\$ 76.396 .917$, que frente al presupuesto de $\$ 65.000 .000$ y sin tener el dato de los CIF reales, arrojan una diferencia de \$11.396.917 (17,5\%), es decir, se sobreaplicaron los CIF presupuestados y con la base de costo de materia prima, los CIF aplicados serían de \$57.785.794, que frente al presupuesto arrojan una diferencia de $\$ 7.214 .206$ (11,1\%), es decir, se subaplicaron los CIF presupuestados.

Si para la distribución de CIF se utiliza una base que combine los dos criterios antes utilizados de manera independiente, el resultado puede mejorar. Para ello y con base en los resultados de la regresión, se le dio un peso de $70 \%$ al criterio de costo materia prima y un peso de $30 \%$ al de los kilos producidos. El cálculo de los CIF aplicados, para la orden 1 con la base combinada sería así:

$(2.000$ kilos $* \$ 1.252,4084 / \mathrm{K}) * 30 \%+$ $(\$ 3.200 .000 * 0.7214) * 70 \%=\$ 2.367 .427$ 
COSTOS INDIRECTOS DE FABRICACIÓN: PROPUESTA PARA SU TRATAMIENTO / B. GUTIÉRREZ, M. DUQUE / 849

\begin{tabular}{cc}
\hline Órdenes & $\begin{array}{c}\text { CIF aplicados base } \\
\text { combinada }\end{array}$ \\
\hline $\mathbf{1}$ & $2.367 .427,33$ \\
\hline $\mathbf{2}$ & $10.322 .153,29$ \\
\hline $\mathbf{3}$ & $12.336 .084,50$ \\
\hline $\mathbf{4}$ & $5.312 .574,98$ \\
\hline $\mathbf{5}$ & $7.225 .507,30$ \\
\hline $\mathbf{6}$ & $6.193 .291,97$ \\
\hline 7 & $8.106 .224,30$ \\
\hline $\mathbf{8}$ & $5.464 .073,32$ \\
\hline $\mathbf{9}$ & $6.041 .793,64$ \\
\hline Total & $\mathbf{6 3 . 3 6 9 . 1 3 0 , 6 4}$ \\
\hline
\end{tabular}

Tabla 9

CIF aplicados con base combinada

Fuente: elaboración propia

Al utilizar una base combinada, los CIF aplicados totales son de $\$ 63.369 .130$, que al compararlos con los $\$ 65.000 .000$ de CIF presupuestados arrojan una variación de $\$ 1.630 .870$ (2,5\%), es decir, se subaplicaron los CIF pero en un valor que se considera razonable.

Manejo contable de la variación: si para el período del ejemplo anterior se suponen unos CIF reales de $\$ 64.000 .000$, el análisis de las variaciones sería el siguiente:

Si se calcula una sola variación: CIF reales - CIF aplicados $=\$ 64.000 .000$ $\$ 63.369 .130=\$ 630.870$ desfavorable

Como se dijo anteriormente, las empresas pueden asignar a resultados o prorratearla entre los inventarios y el costo de ventas. Pero esta variación combina cambios en el dinero gastado y en el nivel de operación que se ejecutó, por tanto y de acuerdo con la NIC 2, deben separarse las dos variaciones que la componen:

\section{Variación de gasto:}

CIF reales - CIF presupuestados $=$ $(\$ 64.000 .000-\$ 65.000 .000)=\$ 1.000 .000 \mathrm{fa}-$ vorable

Esto disminuye el valor de las unidades producidas en el período y debe prorratearse.

$$
\begin{aligned}
& \text { Variación de capacidad: } \\
& (\text { NOR-NOP }) * \text { tasa }=(\$ 80.100 .000- \\
& \$ 90.100 .000) * 0,7214 * 70 \%+(61.000 \mathrm{~K} \\
& -51.900 \mathrm{~K}) * \$ 1.252,4084 * 30 \%=
\end{aligned}
$$

\subsubsection{0,068 desfavorable}

Este valor debe asignarse a resultados, dado que fue una variación relacionada con la capacidad de producción no utilizada.

Este tratamiento implica un manejo contable diferente al que tradicionalmente se les ha venido dando a las variaciones en Colombia. Adicionalmente, el análisis de la variación cambiaría si una parte de los CIF se considera variable.

\section{Conclusiones}

Los CIF son el elemento del costo más complejo de manejar, pues se debe determinar con criterios objetivos la base de actividad con la cual deben distribuirse a los diferentes productos. Una incorrecta definición de la base puede ocasionar una mala asignación de los CIF.

Diferentes estudios han abordado los métodos estadísticos que pueden utilizarse para la separación de los costos de acuerdo con su comportamiento en fijos y variables, pero pocos se han centrado en el análisis de la mejor base de asignación para calcular las tasas de CIF, 
cuando la empresa utiliza sistemas de costeo tradicionales.

Este estudio presenta una propuesta para la definición de la mejor base de asignación de CIF, utilizando para ello un modelo de regresión lineal que permite determinar la base que mayor relación tiene con los costos indirectos, lo cual permitirá a las empresas tener una base matemática que justifique su elección para que no solo se guíen por la intuición o por la experiencia.

Tal como lo muestran los resultados obtenidos al aplicar el modelo, es probable que ninguna de las posibles bases a utilizar tenga un nivel de significancia alto con respecto a los costos indirectos y que su utilización sea riesgosa, como lo han señalado los diferentes autores referenciados anteriormente, mientras que una combinación de varias de ellas puede arrojar un mejor resultado y con ello incrementar la asociación de los CIF a los productos y disminuir las variaciones que puedan presentarse entre los CIF aplicados y los CIF reales cuando la empresa utiliza un sistema de costeo tradicional.

Este estudio solo contempló dos posibles bases de asignación que fueron las que la empresa que sirvió como base para el estudio ha venido calculando y solo se analizaron 16 meses de información. Sería conveniente que en futuros estudios se analicen otras probables bases en un período más amplio de observación.

El análisis del comportamiento de los costos indirectos (fijos y variables) para el cálculo de tasas independientes de CIF, cuando la empresa utiliza un sistema de costeo tradicional, debe estar basado en la variabilidad de los costos en los diferentes períodos y no en su rela- ción con el nivel de operación definido, como se hace para el cálculo de puntos de equilibrio.

La variación de los CIF entre los costos reales y los predeterminados debe separarse en su componente de gasto y de capacidad para que el primero sea asignado como mayor o menor valor de los productos fabricados y/o vendidos y el segundo se asigne a los resultados del período.

\section{Referencias}

Arias-Montoya, Leonel; Portilla de Arias, Liliana Margarita \& Fernández-Henao, Sergio Augusto (2010). La distribución de costos indirectos de fabricación factor clave al costear productos. Scientia et Technica, 16 (45), 79-84. Disponible en: http://www. redalyc.org/articulo.oa?id=84917249014 http://revistas.utp.edu.co/index.php/ revistaciencia/article/view/329/203

Artigas, Jorge Ignacio \& Zayún, Javier Eduardo (2013). Relatividad de los costos. Ponencia presentada en el XXXVI Congreso Argentino de Profesores Universitarios de Costos, Facultad de Ciencias Económicas y Jurídicas de la Universidad Nacional de La Pampa (UNLPam), Santa Rosa, Provincia de La Pampa, Argentina, 2013.

Bendersky, Eduardo (2002). ABC - ABM Gestión de costos por actividades. Buenos Aires: Editorial de las Ciencias. Disponible en: http://www.cosaslibres.com/libro/abc-abmgestion-de-costos-por-actividades_119. html\#

Cartier, Enrique Nicolás (2002). Apuntes para un replanteo de la teoría de los costos fijos. 
Ponencia presentada en el XXV Congreso Argentino de Profesores Universitarios de Costos, Buenos Aires, octubre de 2002.

Colombia (1993). Decreto 2649, por el cual se reglamenta la contabilidad en general y se expiden los principios o normas de contabilidad generalmente aceptados en Colombia, PCGA. Diario Oficial, 41.156, 29 de diciembre de 1993. Disponible en: http://www.actualicese. com/normatividad/2001/decretos/D264993/4D2649-93.htm

Colombia (1993). Decreto 2650, por el cual se modifica el Plan Único de Cuentas, PUC, para los comerciantes. Diario Oficial, 41.156, 29 de diciembre de 1993. Disponible en: http://incp.org.co/Site/2012/ legislativa/2650.pdf

Cuervo-Tafur, Joaquín; Duque-Roldán, María Isabel; Osorio-Agudelo, Jair Albeiro (2013). Costeo Basado en Actividades ABC, Gestión Basada en Actividades ABM. Bogotá: ECOE Editores.

España (2007). Real Decreto 1514, 16 de noviembre de 2007, por el que se aprueba el Plan General de Contabilidad. Boletín Oficial del Estado, BOE, 278, 20 de noviembre de 2007. Disponible en: https:/www.boe.es/ boe/dias/2007/11/20/pdfs/A47402-47407.pdf

Dirección de Impuestos y Aduanas Nacionales, DIAN (1998). Concepto 100447. Disponible en: http://www.ceta.org.co/html/inicio_ de_sesion.asp

Financial Accounting Standards Board, FASB (1953). Accounting Research Bulletins ARB 43: Restatement and Revision of Accounting Research Bulletins. Chapter 4. Disponible en:
http://www.fasb.org/cs/BlobServer?blobkey $=$ id\&blobwhere $=1175820900763 \&$ blobhea der $=$ application $\% 2 F$ pdf\&blobcol=urldata\& blobtable $=$ MungoBlobs

Financial Accounting Standards Board, FASB (2004). Statement of Financial Accounting Standards No. 151: Inventory Costs, an amendment of ARB No. 43, Chapter 4. Disponible en: http://www.fasb.org/cs/BlobSer ver?blobkey $=$ id\&blobwhere $=11758209184$ 58\&blobheader $=$ application\%2Fpdf\&blobc $\mathrm{ol}=$ urldata\&blobtable $=$ MungoBlobs

Horngren, Charles T.; Datar, Srikant M. \& Foster, George (2007). Contabilidad de costos, un enfoque gerencial. México: Editorial Pearson.

Instituto de Contabilidad y Auditoría de Cuentas, ICAC (2000). Resolución ICAC del 9 de mayo de 2000. Disponible en: https://docs.google.com/ document/d/1RIjsqA5WitrmVRc_ To8WnLJ8PikK1zkYI5R0FmdK0Fs/ edit?pli=1, http://www.gabilos.com/ webcontable/icac/090500.htm

International Financial Reporting Standards Foundation, IFRS Foundation \& International Accounting Standards Board, IASB (2011). Normas internacionales de información financiera, NIIF. Reino Unido. Disponible en: http://www.mef.gob.pe/ index.php?option $=$ com_content\&view $=\mathrm{a}$ rticle\&id=2308\&Itemid=101380, http:// www.nicniif.org/home/

International Financial Reporting Standards Foundation, IFRS Foundation \& International Accounting Standards Board, IASB (s.f.). Normas Internacionales de Contabilidad, NIC. Disponible en: http:// 
www.normasinternacionalesdecontabilidad. es/nic/nic.htm

Iudícibus, Sérgio de (1989). Análise de custos. São Paulo: Atlas.

Johnson, H. Thomas \& Kaplan, Robert S. (1988). Ascenso y decadencia de la contabilidad gerencial. Barcelona: Plaza \& Janés Editores.

Kaplan, Alfredo \& Yardín, Amaro (2013). Impacto de los cambios del nivel de actividad en la gestión de los costos. Ponencia presentada en el XXXVI Congreso Argentino de Profesores Universitarios de Costos, Facultad de Ciencias Económicas y Jurídicas de la Universidad Nacional de La Pampa (UNLPam), Santa Rosa, Provincia de La Pampa, Argentina, 2013.

Océano Grupo Editorial (2002). Contabilidad de gestión: presupuestaria y de costos. Madrid: Océano Grupo Editorial.

Rayburn, Letricia Gayle (1999). Contabilidad y administración de costos. México: McGrawHill Interamericana Editores.

Romero-Ceceña, Alfredo (1996). La contabilidad gerencial y los nuevos métodos de costeo. México: Instituto Mexicano de Contadores Públicos, IMCP.
Silva, Felipe Dantas Cassimiro da; Vasconcelos, Marco Túlio de Castro; Silva, Alexandre César Batista da \& Campelo, Sebastião Marcos (2006). Comportamento dos custos: uma investigação empírica acerca dos conceitos econométricos sobre a teoría tradicional da contabilidade de custos. Revista Contabilidade Finançiera, 18 (43), 61-72. Disponible en: http://www.scielo.br/pdf/rcf/v18n43/ a06v1843.pdf

- Fecha de recepción: 25 de junio de 2014

- Fecha de aceptación: 10 de octubre de 2014

- Disponible en línea: 15 de diciembre de 2014

\section{Para citar este artículo}

Gutiérrez-Castañeda, Belky Esperanza \& Duque-Roldán, María Isabel (2014). Costos indirectos de fabricación: propuesta para su tratamiento ante los cambios normativos que enfrenta Colombia [número especial: Contabilidad Gerencial]. Cuadernos de Contabilidad, 15 (39), 829-852. doi: 10.11144/Javeriana.cc15-39.cifp 